\title{
Efficacy of concurrent chemoradiotherapy in subgroups of stage III nasopharyngeal carcinoma: an analysis based on 10-year follow-up
}

Lei Wang ${ }^{1 \dagger}$, Zheng $\mathrm{Wu}^{2+}$, Wanqin Cheng ${ }^{3 \dagger}$, Dehuan Xie ${ }^{5}$, Feifei $\mathrm{Lin}^{4}$, Liangping $\mathrm{Xia}^{{ }^{*}}$ and Yong $\mathrm{Su}^{4^{*}}$ (1)

\begin{abstract}
Purpose: To evaluate the efficacy of concurrent chemoradiotherapy (CCRT) in subgroups of stage III nasopharyngeal carcinoma (NPC) in the context of intensity-modulated radiotherapy (IMRT).

Methods: A total of 272 patients with stage III NPC who underwent IMRT with or without concurrent chemotherapy were retrospectively reviewed. Clinicopathological features were evaluated by a Cox regression model to identify independent prognostic factors. Survival outcomes were assessed using the Kaplan-Meier method and log-rank test.

Results: The median follow-up time was 108 months. The 10-year locoregional-free survival (LRFS), distant metastasis-free survival (DMFS), disease-free survival (DFS), and overall survival (OS) rates were $87.8 \%, 80.7 \%, 68.8 \%$, and 74.9\%, respectively. Multivariate analysis showed that the $\mathrm{N}$ classification was significantly associated with DMFS (hazard ratio [HR] 3.616, 95\% confidence interval [Cl] 1.387-9.428, $P=0.009$ ), DFS (HR 2.417, 95\% Cl 1.291-4.423, $P=0.006)$, and OS (HR 3.024, 95\% Cl 1.385-6.602, $P=0.005)$. In patients with T1-3N2 disease, CCRT was associated with improved 10 -year LRFS (89.6\% vs. $65.4 \%, P=0.005)$, DFS (71.9\% vs. $39.4 \% P=0.001)$ and OS ( $80.0 \%$ vs. $50.5 \%, P=0.004)$ compared with IMRT alone. However, in patients with T3N0-1 disease, no significant survival differences were observed between patients treated with IMRT alone and CCRT $(P>0.05)$.
\end{abstract}

Conclusions: CCRT is an effective therapy in stage III NPC, especially for patients with N2 disease, but IMRT alone may be adequate for N0-1 disease. Individualized treatment strategies are essential for patients with varying disease risks.

\footnotetext{
*Correspondence: xialp@sysucc.org.cn; suyong@sysucc.org.cn

${ }^{\dagger}$ Lei Wang, Zheng Wu, and Wanqin Cheng contributed equally to this work

${ }^{1}$ Department of VIP Region, Sun Yat-sen University Cancer Center, State Key Laboratory of Oncology in South China, Collaborative Innovation Center for Cancer Medicine, Guangdong Key Laboratory of Nasopharyngeal Carcinoma Diagnosis and Therapy, No. 651 Dongfeng Road East, Guangzhou 510060, People's Republic of China

${ }^{4}$ Department of Radiation Oncology, Sun Yat-sen University Cancer Center, State Key Laboratory of Oncology in South China, Collaborative Innovation Center for Cancer Medicine, Guangdong Key Laboratory of Nasopharyngeal Carcinoma Diagnosis and Therapy, No. 651 Dongfeng Road East, Guangzhou 510060, People's Republic of China

Full list of author information is available at the end of the article
}

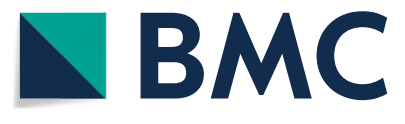

(c) The Author(s) 2021. Open Access This article is licensed under a Creative Commons Attribution 4.0 International License, which permits use, sharing, adaptation, distribution and reproduction in any medium or format, as long as you give appropriate credit to the original author(s) and the source, provide a link to the Creative Commons licence, and indicate if changes were made. The images or other third party material in this article are included in the article's Creative Commons licence, unless indicated otherwise in a credit line to the material. If material is not included in the article's Creative Commons licence and your intended use is not permitted by statutory regulation or exceeds the permitted use, you will need to obtain permission directly from the copyright holder. To view a copy of this licence, visit http://creativecommons.org/licenses/by/4.0/. The Creative Commons Public Domain Dedication waiver (http://creativeco mmons.org/publicdomain/zero/1.0/) applies to the data made available in this article, unless otherwise stated in a credit line to the data. 
Keywords: Stage III, Nasopharyngeal carcinoma, Intensity-modulated radiotherapy, Concurrent chemoradiotherapy, Survival outcome

\section{Introduction}

Nasopharyngeal carcinoma (NPC) is a highly chemoradiosensitive tumor derived from the nasopharyngeal epithelium [1]. Stage I NPC can be treated with intensity-modulated radiotherapy (IMRT) alone, yielding a 5 -year overall survival (OS) rate of more than 90\% [2], while no consensus of treatment strategy for patients with stage II-IVA NPC (based on the 8th TNM staging system [3]) has been established. Thus, the National Comprehensive Cancer Network (NCCN) guidelines recommend patients with stage II-IVA NPC participate in clinical trials with a combination of chemotherapy and radiotherapy to acquire better treatment [4]. However, heterogeneity of the stages results in different survival outcomes, leading to investigations of optimal treatment strategies in different subpopulations.

As higher dose to the target and lower dose to organs at risks (OARs) can be achieved with IMRT compared with two-dimensional conventional radiotherapy (2DCRT) [5], better treatment outcomes have been achieved, especially in stage III NPC [6]. Retrospective studies demonstrated that concurrent chemotherapy (CCT) did not provide survival benefit in stage II and stage T3NOMO NPC but more toxicities in the IMRT era $[7,8]$. Moreover, patients with $\mathrm{N} 1$ stage disease did not acquire therapeutic gain from additional CCT in these studies. Stage III NPC includes disease with T3N0-1 and T1-3N2 stage, and the survival benefit of CCT in heterogeneous disease needs further assessment. To address this issue, we analyzed the long-term survival outcomes in patients with stage III NPC treated with IMRT alone or CCRT among subgroups of T3N0-1 and T1-3N2 disease.

\section{Materials and methods Patients}

Patients with newly-diagnosed nonkeratinizing stage III NPC (according to the 8th TNM staging system) between February 2001 and December 2008 at the Sun Yat-sen University Cancer Center were reviewed. Inclusion criteria were as follows: [1] Karnofsky performance score $\geq 80$; [2] age between 18 and 75 years old; [3] no history of cancer within 5 years; [4] receipt of IMRT; [6] no receipt of neoadjuvant chemotherapy, adjuvant chemotherapy, or target therapy. All clinical records including magnetic resonance imaging (MRI) materials were reviewed. The study was approved by the Medical Ethics Committee of Sun Yat-sen University Cancer Center, and written informed consent was waived due to the retrospective nature. Key data from this study has been uploaded onto the Research Data Deposit public platform (http://www.researchdata.org.cn; approval number: RDDA2019001373).

\section{Radiotherapy}

All patients were treated with IMRT as previously reported. Target volumes and OARs were contoured according to the International Commission on Radiation Units and Measurements Reports 50 and 62 as well as our institutional treatment protocol [9]. Gross tumor volumes (GTVs) included GTVp (the primary gross tumor and metastatic retropharyngeal lymph node) and GTVnd (metastatic cervical lymph node). The clinical target volumes (CTVs) were contoured as CTV1 (highrisk regions: GTVp plus a $5-10 \mathrm{~mm}$ margin and the whole nasopharynx) and CTV2 (low-risk regions: CTV1 plus a $5-10 \mathrm{~mm}$ margin together with the bilateral cervical selective lymph drainage areas). The prescribed doses were 68, 60-66, 60, and 54 Gy in 30 fractions for the planning target volume (PTV) derived from GTVp, GTVnd, CTV1, and CTV2, respectively.

\section{Chemotherapy}

The CCT regimens included [1] cisplatin alone regimen: $80 \mathrm{mg} / \mathrm{m}^{2}$ every three weeks for two to three cycles, or $30 \mathrm{mg} / \mathrm{m}^{2}$ weekly for four to six cycles; [2] PF regimen: combination of cisplatin $\left(80 \mathrm{mg} / \mathrm{m}^{2}\right)$ with 5 -fluorouracil $\left(750 \mathrm{mg} / \mathrm{m}^{2}\right)$ every three weeks for two cycles, or a combination of nedaplatin $\left(80 \mathrm{mg} / \mathrm{m}^{2}\right)$ with 5 -fluorouracil $\left(750 \mathrm{mg} / \mathrm{m}^{2}\right)$ every three weeks for two cycles; and [3] taxol alone regimen: $40 \mathrm{mg} / \mathrm{m}^{2}$ weekly for four to six cycles. Toxicities were evaluated according to the Common Terminology Criteria for Adverse Events (version 3.0).

\section{Follow-up}

Follow-up was measured from the beginning of treatment to the last examination or death. Patients were assessed for the first three months, then every three months for three years, and every 6-12 months thereafter. The endpoints were OS, disease-free survival (DFS), distant metastasis-free survival (DMFS), and locoregional-free survival (LRFS), which referred to the time from treatment to death for any cause; to locoregional failure, distant failure, or death for any cause; to distant failure; and to locoregional failure, respectively. 
Table 1 Baseline characteristics of patients with stage III NPC (N $=272$ )

\begin{tabular}{|c|c|c|c|}
\hline Variables & IMRT alone $(\mathrm{N}=82)$ & CCRT $(N=190)$ & $P$ \\
\hline Sex & & & 0.020 \\
\hline Male & $72(87.8 \%)$ & $143(75.3 \%)$ & \\
\hline Female & $10(12.2 \%)$ & $47(24.7 \%)$ & \\
\hline Age (year) & & & 0.303 \\
\hline$\leq 43$ & 38 (46.3\%) & $101(53.2 \%)$ & \\
\hline$>43$ & $44(53.7 \%)$ & $89(46.8 \%)$ & \\
\hline Smoking & & & 0.070 \\
\hline Yes & $43(52.4 \%)$ & $113(59.5 \%)$ & \\
\hline No & 39 (47.6\%) & 77 (40.5\%) & \\
\hline Alcohol & & & 0.368 \\
\hline Yes & 19 (23.2\%) & $155(81.6 \%)$ & \\
\hline No & $63(76.8 \%)$ & $35(18.4 \%)$ & \\
\hline Tclassification & & & 0.146 \\
\hline $\mathrm{T} 1-2$ & $12(26.8 \%)$ & $36(18.9 \%)$ & \\
\hline T3 & $60(73.2 \%)$ & $154(81.1 \%)$ & \\
\hline Nclassification & & & 0.970 \\
\hline No-1 & 49 (59.8\%) & $114(60.0 \%)$ & \\
\hline N2 & $33(40.2 \%)$ & $76(40.0 \%)$ & \\
\hline EBVDNA & & & 0.398 \\
\hline$<2000$ & 25 (30.5\%) & $68(35.8 \%)$ & \\
\hline$\geq 2000$ & $57(69.5 \%)$ & $122(64.2 \%)$ & \\
\hline
\end{tabular}

NPC, nasopharyngeal carcinoma; IMRT, intensity-modulated radiation therapy; CCRT, concurrent chemoradiotherapy; EBV, Epstein-Barr virus

\section{Statistical methods}

The Mann-Whitney $U$ test was used for ordinal variables, and the chi-squared test was used for categorical variables. The Kaplan-Meier method and log-rank test were used for survival analysis. A Cox regression model was used to identify the independent prognostic factors. Variables were assessed in the univariate analysis, and potential risk factors with statistical significance level of 0.2 were selected for multivariate analysis. SPSS 22.0 software (SPSS Inc., Chicago, IL, USA) and Stata 15.1 (Stata Corporation, College Station, TX, USA) were used for all analyses. A $P$ value of less than 0.05 was considered statistically significant.

\section{Results}

Baseline characteristics

A total of 272 patients meeting the inclusion criteria were included in the study (Table 1). Of the entire cohort, the median age was 43 years old (range 15-75 years old). The distribution of patients between the IMRT alone group and CCRT group was well balanced except for gender $(P$ $=0.020$ ). Of the 272 patients, 190 patients received additional CCT; 132/190 (69.5\%) patients received cisplatin alone, 32/190 (16.8\%) patients received PF, and 26/190 (13.7\%) patients received taxol alone.

\section{Prognostic factors}

Univariate and multivariate analyses (Tables 2 and 3) revealed that the $\mathrm{N}$ classification was significantly associated with DFS (hazard ratio [HR] 1.722, 95\% confidence interval $[\mathrm{CI}] 1.115-2.657, P=0.014)$. CCT was significantly associated with DFS (HR 1.661, 95\% CI 1.064$2.594, P=0.026)$ and OS (HR 1.876, 95\% CI 1.141-3.083, $P=0.013)$. In addition, age (HR 1.946, 95\% CI 1.161$3.264, P=0.012$ ) and smoking (HR 0.578, 95\% CI 0.336$0.996, P=0.048)$ were independent prognostic factors for OS.

\section{Survival outcome}

The median follow-up time was 108 months (range 7-180 months). By the last follow-up, 72 patients had treatment failures, including 20 with locoregional relapse alone (15 with local relapse, 3 with regional relapse, 2 with locoregional relapse), 42 with distant metastasis alone, and 10 with both locoregional relapse and distant metastasis. Moreover, 67 patients had died at the time of

Table 2 Univariate analysis of prognostic factors for 272 patients with stage III NPC

\begin{tabular}{|c|c|c|c|c|c|c|c|c|}
\hline \multirow[t]{2}{*}{ Variables } & \multicolumn{2}{|l|}{ LRFS } & \multicolumn{2}{|l|}{ DMFS } & \multicolumn{2}{|l|}{ DFS } & \multicolumn{2}{|l|}{ OS } \\
\hline & $\mathrm{HR}(95 \% \mathrm{Cl})$ & $P$ & $\mathrm{HR}(95 \% \mathrm{Cl})$ & $P$ & $\mathrm{HR}(95 \% \mathrm{Cl})$ & $P$ & $\mathrm{HR}(95 \% \mathrm{Cl})$ & $P$ \\
\hline Sex (male vs. female) & $0.978(0.400-2.392)$ & 0.961 & $0.922(0.462-1.840)$ & 0.818 & $0.874(0.499-1.530)$ & 0.637 & $0.779(0.407-1.491)$ & 0.451 \\
\hline Age ( $\leq 43$ vs. $>43$, year $)$ & $1.254(0.612-2.570)$ & 0.537 & $1.224(0.706-2.122)$ & 0.471 & $1.450(0.936-2.247)$ & 0.096 & $2.087(1.252-3.480)$ & 0.005 \\
\hline Smoking (yes vs. no) & $0.662(0.323-1.357)$ & 0.260 & $0.618(0.356-1.073)$ & 0.087 & $0.617(0.399-0.953)$ & 0.030 & $0.472(0.286-0.781)$ & 0.003 \\
\hline Alcohol (yes vs. no) & $0.749(0.321-1.746)$ & 0.503 & $0.748(0.392-1.430)$ & 0.380 & $0.707(0.427-1.170)$ & 0.177 & $0.594(0.341-1.035)$ & 0.066 \\
\hline T classification (T1-2 vs. T3) & $1.486(0.371-2.014)$ & 0.735 & $1.580(0.445-1.623)$ & 0.622 & $1.268(0.520-1.451)$ & 0.590 & $1.069(0.581-1.966)$ & 0.830 \\
\hline $\begin{array}{l}\text { N classification (N0-1 vs. } \\
\text { N2) }\end{array}$ & $1.890(0.923-3.873)$ & 0.082 & $1.672(0.965-2.895)$ & 0.067 & $1.670(1.083-2.575)$ & 0.020 & $1.506(0.922-2.459)$ & 0.102 \\
\hline СCT (yes vs. no) & $1.721(0.828-3.575)$ & 0.146 & $1.485(0.842-2.620)$ & 0.172 & $1.721(1.106-2.678)$ & 0.016 & $1.976(1.205-3.239)$ & 0.007 \\
\hline EBV DNA (<2000 vs. $\geq 2000)$ & $1.747(0.750-4.071)$ & 0.196 & $1.398(0.756-2.585)$ & 0.286 & $1.084(0.684-1.718)$ & 0.732 & $1.081(0.649-1.802)$ & 0.764 \\
\hline
\end{tabular}

NPC, nasopharyngeal carcinoma; HR, hazard ratio; Cl, confidence interval; LRFS, locoregional-free survival; DMFS, distant metastasis-free survival; DFS, disease-free survival; OS, overall survival; CCT, concurrent chemotherapy; EBV, Epstein-Barr virus 
Table 3 Multivariate analysis of prognostic factors for 272 patients with stage III NPC

\begin{tabular}{|c|c|c|c|c|c|c|c|c|}
\hline \multirow[t]{2}{*}{ Variables } & \multicolumn{2}{|l|}{ LRFS } & \multicolumn{2}{|l|}{ DMFS } & \multicolumn{2}{|l|}{ DFS } & \multicolumn{2}{|l|}{ OS } \\
\hline & $\mathrm{HR}(95 \% \mathrm{Cl})$ & $P$ & $\mathrm{HR}(95 \% \mathrm{Cl})$ & $P$ & HR $(95 \% \mathrm{Cl})$ & $P$ & $\mathrm{HR}(95 \% \mathrm{Cl})$ & $P$ \\
\hline Age ( $\leq 43$ vs. $>43$, year) & - & - & - & - & $1.378(0.884-2.148)$ & 0.157 & $1.946(1.161-3.264)$ & 0.012 \\
\hline Smoking (yes vs. no) & - & - & - & - & $0.728(0.452-1.172)$ & 0.192 & $0.578(0.336-0.996)$ & 0.048 \\
\hline Alcohol (yes vs. no) & - & - & $0.643(0.369-1.121)$ & 0.120 & $0.872(0.506-1.502)$ & 0.621 & $0.839(0.462-1.522)$ & 0.563 \\
\hline N classification (N0-1 vs. N2) & $1.910(0.930-3.920)$ & 0.078 & $1.683(0.972-2.916)$ & 0.063 & $1.722(1.115-2.657)$ & 0.014 & $1.629(0.996-2.665)$ & 0.052 \\
\hline CCT (yes vs. no) & $1.737(0.834-3.617)$ & 0.140 & $1.431(0.808-2.535)$ & 0.219 & $1.661(1.064-2.594)$ & 0.026 & $1.876(1.141-3.083)$ & 0.013 \\
\hline EBV DNA (<2000 vs. $\geq 2000)$ & $1.664(0.713-3.883)$ & 0.239 & - & - & - & - & - & - \\
\hline
\end{tabular}

NPC, nasopharyngeal carcinoma; HR, hazard ratio; Cl, confidence interval; LRFS, locoregional-free survival; DMFS, distant metastasis-free survival; DFS, disease-free survival; OS, overall survival; CCT, concurrent chemotherapy; EBV, Epstein-Barr virus

the last follow-up. Specifically, 44 died of distant metastasis alone, 7 died of locoregional relapse alone, 3 died of nasopharyngeal hemorrhage, and 13 died of other reasons (i.e., accidents). Of the entire cohort, the 10-year LRFS, DMFS, DFS, and OS were $87.8 \%, 80.7 \%, 68.8 \%$, and $74.9 \%$, respectively. Significant differences were observed between individuals treated with IMRT alone and CCRT in 10 -year OS $(63.8 \%$ vs. $80.1 \%, P=0.006)$ and DFS (58.6\% vs. $73.6 \%, P=0.014$ ), while no significant differences were observed in 10 -year LRFS ( $83.3 \%$ vs. $89.6 \%, P$ $=0.140)$ and DMFS $(75.7 \%$ vs. $82.8 \%, P=0.169$, Fig. 1$)$.

We also evaluated survival outcomes in the low-risk group (stage T3N0-1, $\mathrm{n}=163$ ) and the high-risk group (stage T1-3N2, $\mathrm{n}=109$ ) when treated with IMRT alone or CCRT. In the low-risk group, the baseline characteristics were well balanced between the IMRT alone group and CCRT group except for sex $(P=0.037)$ (Additional file 1: Table S1). No significant survival differences were observed between patients who received IMRT alone $(\mathrm{n}=49)$ and CCRT $(\mathrm{n}=114)$, including LRFS, DMFS, DFS, and OS $(P>0.05$, Fig. 2$)$. In the high-risk group, the baseline characteristics were well balanced between the IMRT alone group and CCRT group except for alcohol consumption $(P=0.040)$ (Additional file 1: Table S1). Significant survival differences were observed between patients who received IMRT alone $(\mathrm{n}=33)$ and CCRT $(\mathrm{n}$ $=76$ ), including LRFS (65.4\% vs. $89.6 \%, P=0.005)$, DFS (39.4\% vs. $71.9 \%, P=0.001)$, and OS $(50.5 \%$ vs. $80.0 \%$, $P=0.002)$. Interestingly, no significant difference was observed in DMFS (65.4\% vs. $80.0 \%, P=0.091)$ between patients who received IMRT alone and CCRT in the high-risk group (Fig. 3).

\section{Toxicity}

The incidences of Grade 3-4 acute adverse events (AEs) in 272 patients are listed in Table 4. Patients treated with CCRT experienced more hematological AEs, including leukopenia ( $11.6 \%$ vs. $1.2 \%, P=0.005)$, neutropenia (7.4\% vs. $0.0 \%, P=0.012$ ), and thrombocytopenia $(4.8 \%$ vs. $0.0 \%, P=0.045)$, than those treated with IMRT alone except for anemia ( $2.1 \%$ vs. $0.0 \%, P=0.186)$. Moreover, a higher incidence of mucositis was observed in the CCRT group compared with the IMRT alone group $(50.5 \%$ vs. $19.5 \%, P<0.001$ ), while no statistical differences were observed in xerostomia, dermatitis, nausea/vomiting, and hepatoxicity between the two groups $(P>0.05)$.

\section{Discussion}

A previous meta-analysis showed that CCT brought survival benefit to patients with locoregionally advanced NPC in the context of 2DCRT, even in patients with stage II disease [10]. Thus, radiotherapy combined with chemotherapy has been guideline-recommended in NPC except for stage I disease [4], since additional CCT is regarded as the main contributor to improved radiosensitivity. However, the development of IMRT, which allows sharper radiation dose gradients between tumor and critical organs, provides improved locoregional control and reduced toxicities in the treatment of NPC [6]. Therefore, it is necessary to re-assess the relative gain of additional CCT in heterogeneous disease treated with IMRT.

Investigators have reported the efficacy of CCRT in NPC in the IMRT era with controversial results. Sun et al. [11] and Yi et al. [12] did not observe survival improvement in locoregionally advanced NPC when treated with CCRT, while researchers from Hong Kong observed enhanced treatment outcomes in NPC with additional CCT of cisplatin [13]. This inconsistency might result from the heterogeneity of enrolled patients, who were staged at II-IV. Pan and colleagues [14] found similar survival outcomes between the IMRT alone group and CCRT group $(P>0.05)$ in stage II NPC, indicating that the benefit of additional CCT might contribute little to the already excellent locoregional control. Moreover, Zhang et al. [7] failed to identify CCT as an independent prognostic factor in stage T3NOMO and stage II NPC, and subgroup analysis revealed that $\mathrm{CCT}$ did not provide a significant survival 


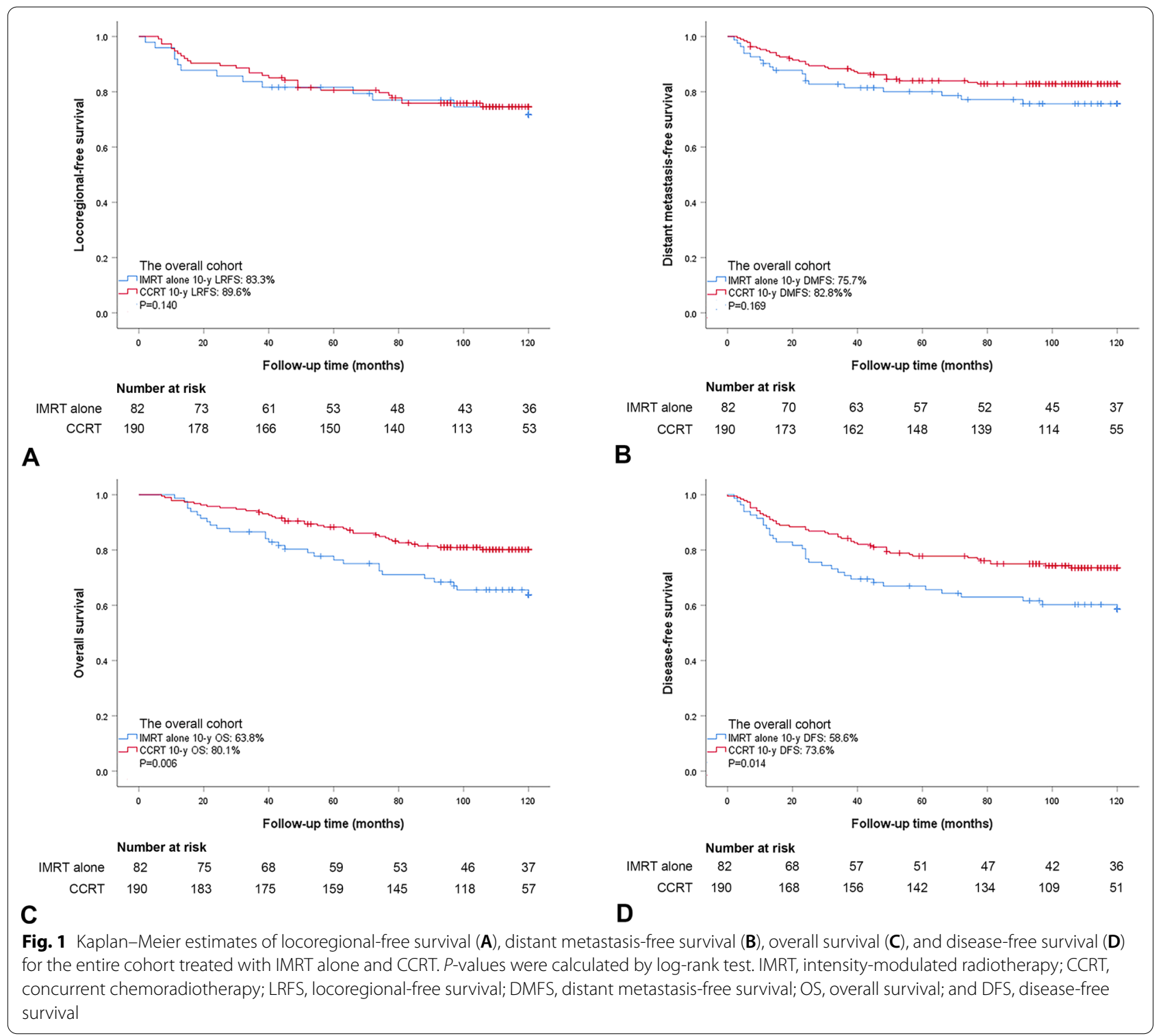

difference in patients with N1 disease $(P>0.05)$. Similar results were observed by Aftab and colleagues [8]. Thus, although the combination of radiotherapy and chemotherapy is recommended in stage III NPC, the role of CCT will require further assessment.

To the best of our knowledge, this is the first comparison study evaluating IMRT alone and CCRT in stage III NPC. In the multivariate analysis, N classification was the main independent prognostic factor for DFS (HR 1.722, 95\% CI 1.115-2.657, $P=0.014$ ). Of the entire cohort, 10 -year OS (63.8\% vs. $80.1 \% ; P=0.006)$ and DFS $(58.6 \%$ vs. $73.6 \% ; P=0.014)$ were better in the CCRT group compared with the IMRT alone group. This indicated that patients with stage III disease could benefit from additional CCT, which is consistent with previous studies of locoregionally advanced cases [15, 16]. However, when we divided patients into a low-risk group of staged T3N0-1disease and high-risk group of staged T1-3N2 disease to address this issue, the survival differences were not statistically significant when patients were treated with IMRT alone and CCRT, including OS, DFS, DMFS, and LRFS $(P>0.05)$, in the low-risk subgroup. By contrast, in the high-risk group, the 10 -year LRFS $(65.4 \%$ vs. $89.6 \%, P=0.005)$, DFS (39.4\% vs. $71.9 \%, P=0.001)$, and OS $(50.5 \%$ vs. $80.0 \%$, $P=0.002)$ were significantly better when patients were treated with CCRT rather than IMRT alone except for DMFS (65.4\% vs. $80.0 \%, P=0.091)$. 


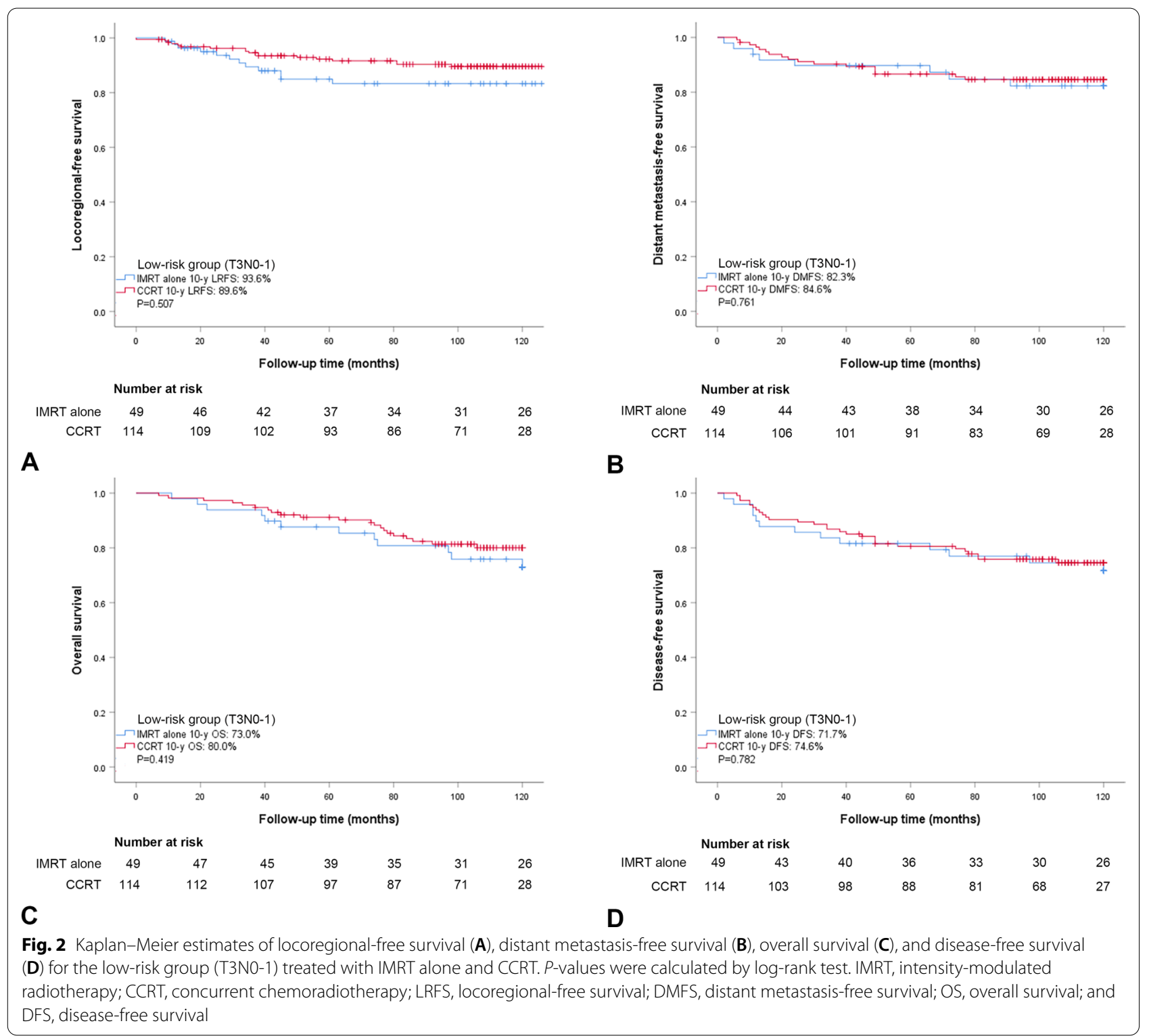

There are several potential explanations for our findings. Firstly, the benefit of locoregional control from additional CCT might need to be re-assessed in the IMRT era. In the present study, comparable LRFS was observed in patients treated with IMRT alone and CCRT (93.6\% vs. $89.6 \%, P=0.507)$ in the low-risk group. We propose that the substantial benefit of CCT might be narrowed with application of IMRT in disease with low tumor burden. By contrast, worse LRFS was observed in patients treated with IMRT alone than CCRT ( $65.4 \%$ vs. $89.6 \%, P=0.005)$ in the high-risk group, indicating that additional CCT might still be effective in patients with high tumor burden because of its ability to improve radiosensitivity.
Secondly, the present study failed to identify significant improvement in DMFS in the whole cohort comparing CCRT with IMRT alone $(82.8 \%$ vs. $75.7 \%, P=0.169)$. Although there was a trend of better DMFS in the highrisk group when treated with CCRT (IMRT alone vs. CCRT: $65.4 \%$ vs. $80.0 \%, P=0.091$ ), statistical significance was not observed. Further explorations of combination therapy for patients in the high-risk group are expected to reduce distant metastasis.

Thirdly, individualized treatment strategies need to be planned based on stratified risk of locoregional failure and distant failure. In clinical practice, stage IIIIVA NPC disease is regarded as locoregional advanced NPC, and similar treatments are recommended [4]. 


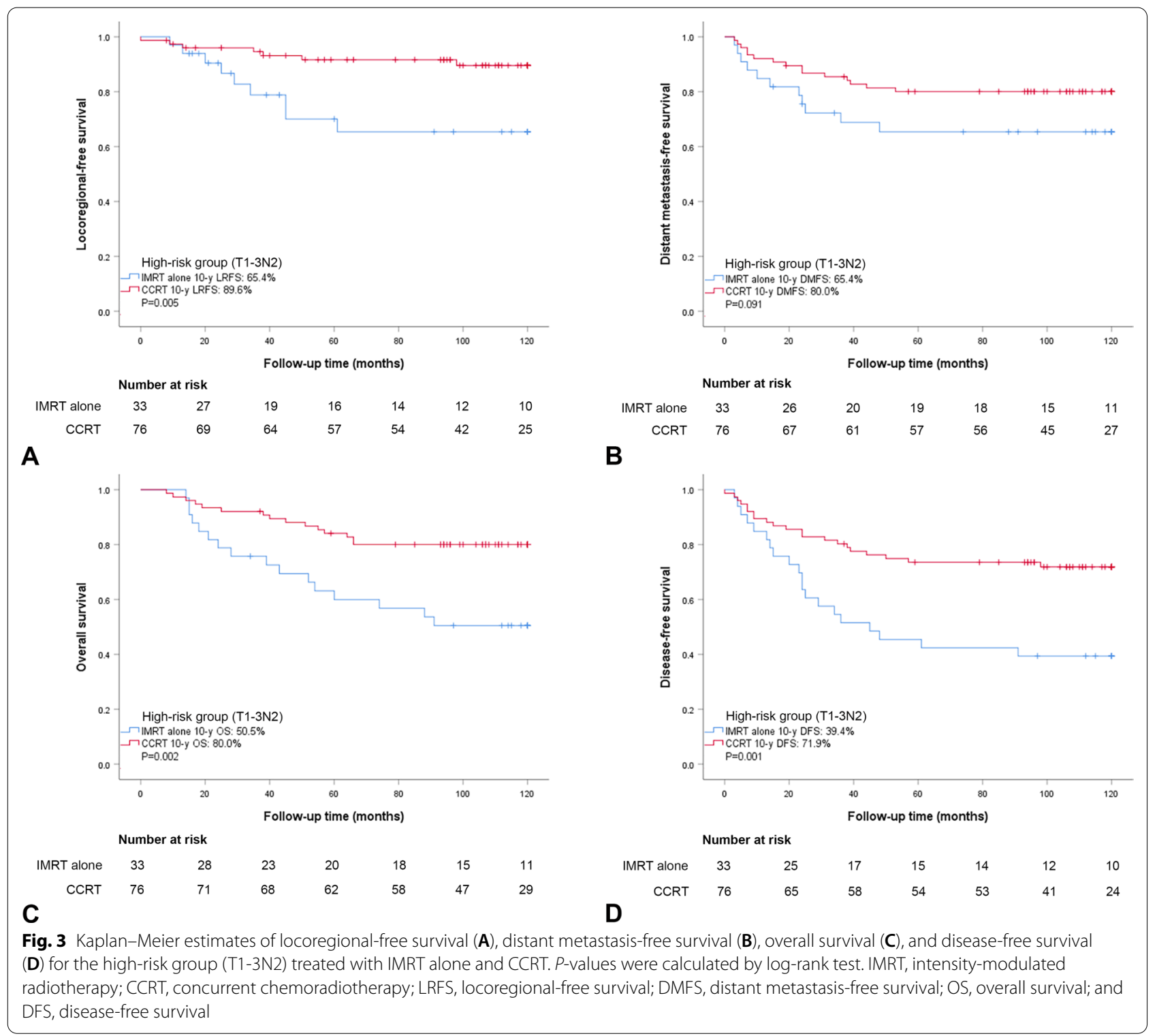

However, previous studies demonstrated that prognosis of patients with stage III NPC was better than that of patients with stage IVA NPC [3]. Moreover, investigators reported no significant survival difference between the stage T3N0M0 subgroup and stage II subgroup, and IMRT alone was effective [7]. Thus, the treatment failure risk in stage III disease varies. Consistent with previous results [17], the present study revealed that stage III NPC treated with IMRT had different risk of treatment failures depending on the $\mathrm{N}$ stage. Therefore, if similar treatment strategies are provided for stage III disease, intensive treatment might be an aggressive approach in the low-risk group, while patients in the high-risk group might receive insufficient treatment.
On the other hand, increased Grade 3-4 hematological AEs were observed in the CCRT group compared with the IMRT alone group $(P<0.05)$ except for anemia $(P=0.186)$. Moreover, the incidence rate of Grade 3-4 mucositis was higher in the CCRT group compared with the IMRT alone group $(P<0.001)$. We postulated that the differences might result from the additional CCT in the CCRT group. However, no significant differences were observed between the two treatment groups regarding other non-hematological Grade 3-4 AEs such as xerostomia and dermatitis. We attributed the similar incidence of radiotherapy-related AEs in the two groups to the advanced IMRT techniques. Therefore, additional CCT might not provide significant survival benefit but 
Table 4 Grade 3-4 acute adverse events in 272 patients with stage III NPC

\begin{tabular}{lccc}
\hline Variables & IMRT (N= 82) & CCRT (N= 190) & $P$ \\
\hline Hematological & & & \\
Leukopenia & $1(1.2 \%)$ & $22(11.6 \%)$ & 0.005 \\
Neutropenia & $0(0.0 \%)$ & $14(7.4 \%)$ & 0.012 \\
Anemia & $0(0.0 \%)$ & $4(2.1 \%)$ & 0.186 \\
Thrombocytopenia & $0(0.0 \%)$ & $9(4.8 \%)$ & 0.045 \\
Non-hematological & & & \\
Mucositis & $16(19.5 \%)$ & $96(50.5 \%)$ & $<0.001$ \\
Xerostomia & $0(0.0 \%)$ & $4(2.1 \%)$ & 0.186 \\
Dermatitis & $3(3.7)$ & $6(3.2 \%)$ & 0.833 \\
Nausea/vomiting & $0(0.0 \%)$ & $5(2.6 \%)$ & 0.139 \\
Hepatoxicity & $1(1.2 \%)$ & $0(0.0 \%)$ & 0.128 \\
\hline
\end{tabular}

NPC, nasopharyngeal carcinoma; IMRT, intensity-modulated radiation therapy; CCRT, concurrent chemoradiotherapy

increased Grade 3-4 hematological toxicities in the lowrisk group, and the routine use of CCT in this subgroup needs further evaluation.

There are several limitations of this study. This is a retrospective study with a small sample size. The IMRT technique was introduced in our institution in 2001. Therefore, the number of patients with stage III disease who received IMRT between 2001 and 2008 is limited. The efficacy of CCT in locoregionally advanced NPC achieved a consensus in 2006 based on two meta-analyses $[10,18]$. Therefore, the treatment strategy for patients with stage III disease was not uniform before 2008, and rare patients with stage III disease received IMRT alone after 2008 in our institution. However, the data during those years is valuable, and allowed us to conduct the current retrospective study assessing the efficacy of CCT in subgroups of stage III disease. Moreover, the dataset in the present study was from the Asian population, in which NPC is prevalent with a high rate of Epstein-Barr virus (EBV) infection. The validity of the current findings in non-endemic, non-EBV related regions needs further exploration. Of note, this study was based on long-term follow-up of real-world data, with a focus on a special subpopulation of NPC. Therefore, our results provide a basis for future research of individualized treatment modalities for stage III NPC.

\section{Conclusions}

Our results demonstrated that CCRT was able to improve survival outcomes in stage III NPC, especially for patients with $\mathrm{N} 2$ disease, while survival benefit of additional CCT in low-risk subgroup with N0-1 disease was limited with increased toxicities. Further randomized trials are warranted to confirm the results of our study.

\begin{abstract}
Abbreviations
NPC: Nasopharyngeal carcinoma; IMRT: Intensity-modulated therapy; 2DCRT :Two-dimensional conventional radiotherapy; CCRT: Concurrent chemoradiotherapy; CCT: Concurrent chemotherapy; HR: Hazard ratio; Cl: Confidence interval; NCCN: National Comprehensive Cancer Network; MRI: Magnetic resonance imaging; OS: Overall survival; LRFS: Locoregional recurrence-free survival; DMFS: Distant metastasis-free survival; DFS: Disease-free survival; OARs: Organs at risk; GTV: Gross tumor volume; GTVp: Primary gross tumor (including retropharyngeal lymph node metastases); GTVnd: Cervical lymph node metastasis; CTV1: High-risk clinical target volume; CTV2: Low-risk clinical target volume; PTV: Planning target volume; PF: Combination of cisplatin (80 $\mathrm{mg} / \mathrm{m}^{2}$ ) with 5 -fluorouracil $\left(750 \mathrm{mg} / \mathrm{m}^{2}\right)$, or combination of nedaplatin (80 $\left.\mathrm{mg} / \mathrm{m}^{2}\right)$ with 5-fluorouracil $\left(750 \mathrm{mg} / \mathrm{m}^{2}\right)$; AE: Acute adverse event.
\end{abstract}

\section{Supplementary Information}

The online version contains supplementary material available at https://doi. org/10.1186/s13014-021-01929-9.

Additional file 1. Table S1. Baseline characteristics of patients with stage III NPC in N0-1 and N2 subgroups.

\section{Acknowledgements}

The authors declare that they have no funding.

\section{Authors' contributions}

LX, YS: conception and design; LW, ZW, WC: data collection, statistical analysis; LW, DX: manuscript preparation; ZW, WC, FL: manuscript editing; LX, YS: quality control of data and manuscript review. All authors read and approved the final manuscript.

\section{Availability of data and materials}

All data generated or analyzed during this study are included in this published article.

\section{Declarations}

Ethics approval and consent to participate

This study was approved by the Medical Ethics Committee of Affiliated Hospital of Sun Yat-sen University Cancer Center, and the need for written informed consent was waived.

\section{Consent for publication}

Not applicable.

\section{Competing interests}

The authors declare that they have no competing interests.

\section{Author details}

${ }^{1}$ Department of VIP Region, Sun Yat-sen University Cancer Center, State Key Laboratory of Oncology in South China, Collaborative Innovation Center for Cancer Medicine, Guangdong Key Laboratory of Nasopharyngeal Carcinoma Diagnosis and Therapy, No. 651 Dongfeng Road East, Guangzhou 510060, People's Republic of China. ${ }^{2}$ Department of Radiation Oncology, Hunan Cancer Hospital and The Affiliated Cancer Hospital of Xiangya School of Medicine, Central South University, 283 Tong Zi Po Road, Changsha 410013, People's Republic of China. ${ }^{3}$ Department of Radiation Oncology, Shunde Hospital of Southern Medical University, Foshan 528399, People's Republic of China. ${ }^{4}$ Department of Radiation Oncology, Sun Yat-sen University Cancer Center, State Key Laboratory of Oncology in South China, Collaborative Innovation Center for Cancer Medicine, Guangdong Key Laboratory of Nasopharyngeal Carcinoma Diagnosis and Therapy, No. 651 Dongfeng Road East, Guangzhou 510060, People's Republic of China. ${ }^{5}$ Department of Radiation Nasopharyngeal Carcinoma, Sun Yat-sen University Cancer Center, State Key Laboratory of Oncology in South China, Collaborative Innovation Center for Cancer Medicine, Guangdong Key Laboratory of Nasopharyngeal 
Carcinoma Diagnosis and Therapy, No. 651 Dongfeng Road East, Guangzhou 510060, People's Republic of China.

Received: 10 July 2021 Accepted: 9 October 2021

Published online: 06 November 2021

\section{References}

1. Chen YP, Chan ATC, Le QT, Blanchard P, Sun Y, Ma J. Nasopharyngeal carcinoma. Lancet. 2019;394(10192):64-80.

2. Su SF, Han F, Zhao C, Chen CY, Xiao WW, Li JX, et al. Long-term outcomes of early-stage nasopharyngeal carcinoma patients treated with intensity-modulated radiotherapy alone. Int J Radiat Oncol Biol Phys. 2012:82(1):327-33.

3. Pan JJ, Ng WT, Zong JF, Chan LL, O'Sullivan B, Lin SJ, et al. Proposal for the 8th edition of the AJCC/UICC staging system for nasopharyngeal cancer in the era of intensity-modulated radiotherapy. Cancer. 2016;122(4):546-58.

4. Pfister DG, Spencer S, Adelstein D, Adkins D, Brizel DM, Bruce JY, et al. NCCN clinical practice guidelines in oncology (NCCN Guidelines ${ }^{\circledR}$ ) Head and Neck Cancers. Version 3. 2021.

5. Lee N, Xia P, Quivey JM, Sultanem K, Poon I, Akazawa C, et al. Intensitymodulated radiotherapy in the treatment of nasopharyngeal carcinoma: an update of the UCSF experience. Int J Radiat Oncol Biol Phys. 2002;53(1):12-22.

6. Peng G, Wang T, Yang KY, Zhang S, Zhang T, Li Q, et al. A prospective, randomized study comparing outcomes and toxicities of intensitymodulated radiotherapy vs. conventional two-dimensional radiotherapy for the treatment of nasopharyngeal carcinoma. Radiother Oncol. 2012;104(3):286-93.

7. Zhang F, Zhang Y, Li WF, Liu X, Guo R, Sun Y, et al. Efficacy of concurrent chemotherapy for intermediate risk NPC in the intensity-modulated radiotherapy era: a propensity-matched analysis. Sci Rep. 2015;5:17378.

8. Aftab O, Liao S, Zhang R, Tang N, Luo M, Zhang B, et al. Efficacy and safety of intensity-modulated radiotherapy alone versus intensity-modulated radiotherapy plus chemotherapy for treatment of intermediate-risk nasopharyngeal carcinoma. Radiat Oncol. 2020;15(1):66.

9. Lai SZ, Li WF, Chen L, Luo W, Chen YY, Liu LZ, et al. How does intensitymodulated radiotherapy versus conventional two-dimensional radiotherapy influence the treatment results in nasopharyngeal carcinoma patients? Int J Radiat Oncol Biol Phys. 2011;80(3):661-8.
10. Langendijk JA, Leemans CR, Buter J, Berkhof J, Slotman BJ. The additional value of chemotherapy to radiotherapy in locally advanced nasopharyngeal carcinoma: a meta-analysis of the published literature. J Clin Oncol. 2004;22(22):4604-12.

11. Sun X, Su S, Chen C, Han F, Zhao C, Xiao W, et al. Long-term outcomes of intensity-modulated radiotherapy for 868 patients with nasopharyngeal carcinoma: an analysis of survival and treatment toxicities. Radiother Oncol. 2014;110(3):398-403.

12. Yi J, Huang $X$, Gao L, Luo J, Zhang S, Wang K, et al. Intensity-modulated radiotherapy with simultaneous integrated boost for locoregionally advanced nasopharyngeal carcinoma. Radiat Oncol. 2014;9:56.

13. Au KH, Ngan RKC, Ng AWY, Poon DMC, Ng WT, Yuen KT, et al. Treatment outcomes of nasopharyngeal carcinoma in modern era after intensity modulated radiotherapy (IMRT) in Hong Kong: a report of 3328 patients (HKNPCSG 1301 study). Oral Oncol. 2018;77:16-21.

14. Pan XB, Li L, Qu S, Chen L, Liang SX, Zhu XD. The efficacy of chemotherapy in survival of stage II nasopharyngeal carcinoma. Oral Oncol. 2020;101:104520.

15. Chan AT, Leung SF, Ngan RK, Teo PM, Lau WH, Kwan WH, et al. Overall survival after concurrent cisplatin-radiotherapy compared with radiotherapy alone in locoregionally advanced nasopharyngeal carcinoma. J Natl Cancer Inst. 2005;97(7):536-9.

16. Chan AT, Teo PM, Ngan RK, Leung TW, Lau WH, Zee B, et al. Concurrent chemotherapy-radiotherapy compared with radiotherapy alone in locoregionally advanced nasopharyngeal carcinoma: progressionfree survival analysis of a phase III randomized trial. J Clin Oncol. 2002;20(8):2038-44.

17. Wu LR, Zhang XM, Xie XD, Lu Y, Wu JF, He X. Validation of the 8th edition of AJCC/UICC staging system for nasopharyngeal carcinoma: results from a non-endemic cohort with 10-year follow-up. Oral Oncol. 2019;98:141-6.

18. Huncharek M, Kupelnick B. Combined chemoradiation versus radiation therapy alone in locally advanced nasopharyngeal carcinoma: results of a meta-analysis of 1528 patients from six randomized trials. Am J Clin Oncol. 2002;25(3):219-23.

\section{Publisher's Note}

Springer Nature remains neutral with regard to jurisdictional claims in published maps and institutional affiliations.
Ready to submit your research? Choose BMC and benefit from:

- fast, convenient online submission

- thorough peer review by experienced researchers in your field

- rapid publication on acceptance

- support for research data, including large and complex data types

- gold Open Access which fosters wider collaboration and increased citations

- maximum visibility for your research: over 100M website views per year

At BMC, research is always in progress.

Learn more biomedcentral.com/submissions 